\title{
Perturbações do Comportamento Alimentar na adolescência
}

Helena Fonseca*

\section{RESUMO}

O objectivo deste artigo é o de examinar o modo como a epidemiologia, o diagnóstico, as complicações médicas e nutricionais, as questões psicológicas, o tratamento e o prognóstico dos adolescentes com uma Perturbação do Comportamento Alimentar diferem dos dos adultos, sobretudo no que respeita à prevenção e às questões ligadas com o desenvolvimento que caracterizam este período.

Palavras-chave: Perturbação do Comportamento Alimentar; Adolescente; Prevenção.

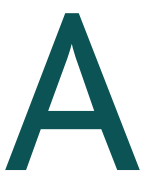
etiologia das Perturbações do Comportamento Alimentar é multifactorial. Na nossa sociedade ocidental a prevalência estimada da Anorexia Nervosa é de $0,4 \%$ e da Bulimia Nervosa de $1 \%$. Constituem factores de risco na adolescência, o sexo feminino, as dietas repetidas, o temperamento perfeccionista, ser um maturador precoce, ter sido alvo de comentários desagradáveis relacionados com o peso, baixa auto-estima, história de lutos ou perdas, disfunção familiar.

A adolescência é caracterizada por um grande leque de transformações a nível biológico, cognitivo e psicossocial. ${ }^{1}$ Estas transformações processam-se a um ritmo muito rápido, gerando frequentemente perplexidade e, por vezes, alguma ansiedade ao adolescente. As transformações pubertárias acompanham-se de alterações na composição corporal que vão introduzir diferenças marcadas entre géneros. Enquanto no sexo feminino a percentagem de massa gorda aumenta, quase duplicando entre os estádios 1 e 5 de Tanner (Quadro I), no sexo masculino diminui, havendo em contrapartida aumento da massa muscular. No sexo feminino, para além do aumento da percentagem de massa gorda, vai ocorrer um alargamento da bacia que só mais tarde vai aumentar no seu diâmetro ântero-posterior. ${ }^{2}$ Muito frequente-

*Chefe de Serviço, Unidade de Medicina do Adolescente, Departamento da Criança e da Família, Hospital de Santa Maria. mente, a adolescente interpreta estas transformações como tendo excesso de peso ou mesmo obesidade.

A Consulta de Vigilância de Saúde constitui uma excelente oportunidade para abordar estas questões e prestar assim cuidados antecipatórios. Se esta oportunidade falha, pode originar-se uma insatisfação com a imagem corporal, que poderá conduzir a uma Perturbação do Comportamento Alimentar (PCA) em personalidades específicas ou quando estão presentes outros factores de risco. A avaliação da dinâmica familiar é também importante, dado que pode ser protectora ou,

\begin{tabular}{l}
$\begin{array}{l}\text { QUADRO I. Percentagem de massa gorda durante a } \\
\text { puberdade (adaptado de ref. } 2 \text { ) }\end{array}$ \\
\begin{tabular}{cc} 
Estádio pubertário & \% de massa gorda \\
\hline Sexo Feminino & 15,7 \\
\hline 1 & 18,9 \\
\hline 2 & 21,6 \\
\hline 3 & 26,7 \\
\hline 4 & 14,3 \\
\hline Sexo masculino & 11,2 \\
\hline 1 & \\
\hline 2 &
\end{tabular} \\
\hline $\begin{array}{l}\text { Nos estádios 3, } 4 \text { e } 5 \text { a percentagem de massa gorda } \\
\text { mantém-se constante }\end{array}$
\end{tabular}


pelo contrário, indutora de disfunção. A presença materna, por exemplo, foi identificada como um factor protector contra comportamentos extremos de controlo do peso, para ambos os sexos. ${ }^{3}$

A inclusão da avaliação da imagem corporal, através da utilização, por exemplo, do diagrama de Stunkard (Figura 1) pode ser muito informativa, ${ }^{4}$ assim como a inclusão de três questões: "Como te vês?», «Como achas quer os outros te vêem?»; «Como gostarias de ser?».

A má interpretação dos fenómenos que estão a acontecer ou o descontentamento com a imagem corporal pode levar o adolescente à adopção de dietas auto-prescritas com o objectivo de perder peso (mesmo na ausência de excesso de peso) ou mesmo a medidas extremas de controlo do peso, estas mais frequentes na população obesa. ${ }^{5}$ Nas várias versões do Estudo Health Behaviour in School-Aged Children realizado em Portugal, cerca de 7\% dos adolescentes inquiridos reportaram estar a fazer dieta com o objectivo de perder peso e quase $1 / 4$ dos adolescentes referiu não estar a fazer dieta mas considerar que necessitava de perder peso. ${ }^{6}$ Estar a fazer dieta foi consistentemente mais frequente no sexo feminino enos mais velhos. Entre os que referiram estar a fazer dieta, cerca de $60 \%$ eram normoponderais, ${ }^{7}$ o que é preocupante, dado que a adopção de sucessivos planos de dieta constitui por si só, como se sabe, um factor de risco de PCA. ${ }^{8}$

Uma dieta restritiva pode começar gradualmente num contexto de preocupação crescente com o corpo e os alimentos.

Deverão constituir sinais de alerta para o médico de família ou pediatra:

- Pedidos insistentes de dietas

- Fixação num peso ideal

- Obsessão com o conteúdo calórico dos alimentos

- Exercício físico intenso para controlar o peso

- Irregularidades menstruais

- Pedidos regulares de:

- Laxantes

- Diuréticos

- Supressores do apetite

- Isolamento da família às refeições

\section{ANOREXIA NERVOSA - CRITÉRIOS DIAGNÓSTICOS (DSM IV-TR) ${ }^{9}$}

A. Recusa em manter um peso corporal igual ou superior ao minimamente normal para a idade e altura (por exemplo, perda de peso que leva a manter um peso inferior a $85 \%$ do esperado; ou incapacidade em ganhar o peso esperado para o crescimento, ficando aquém de $85 \%$ do previsto).

B. Medo intenso de ganhar peso ou de engordar, mesmo quando o peso é insuficiente.

C. Perturbação na apreciação do peso e forma corporal, indevida influência do peso e forma corporal na auto-avaliação, ou negação da gravidade do grande emagrecimento actual.

D. Nas jovens após a menarca, amenorreia, ou seja, ausência de pelo menos três ciclos menstruais consecutivos (considera-se que uma mulher tem amenorreia se a menstruação ocorrer somente após administração de hormonas, por exemplo, estrógenos).

Especificação do tipo:

- Tipo Restritivo: durante o episódio actual de Ano-

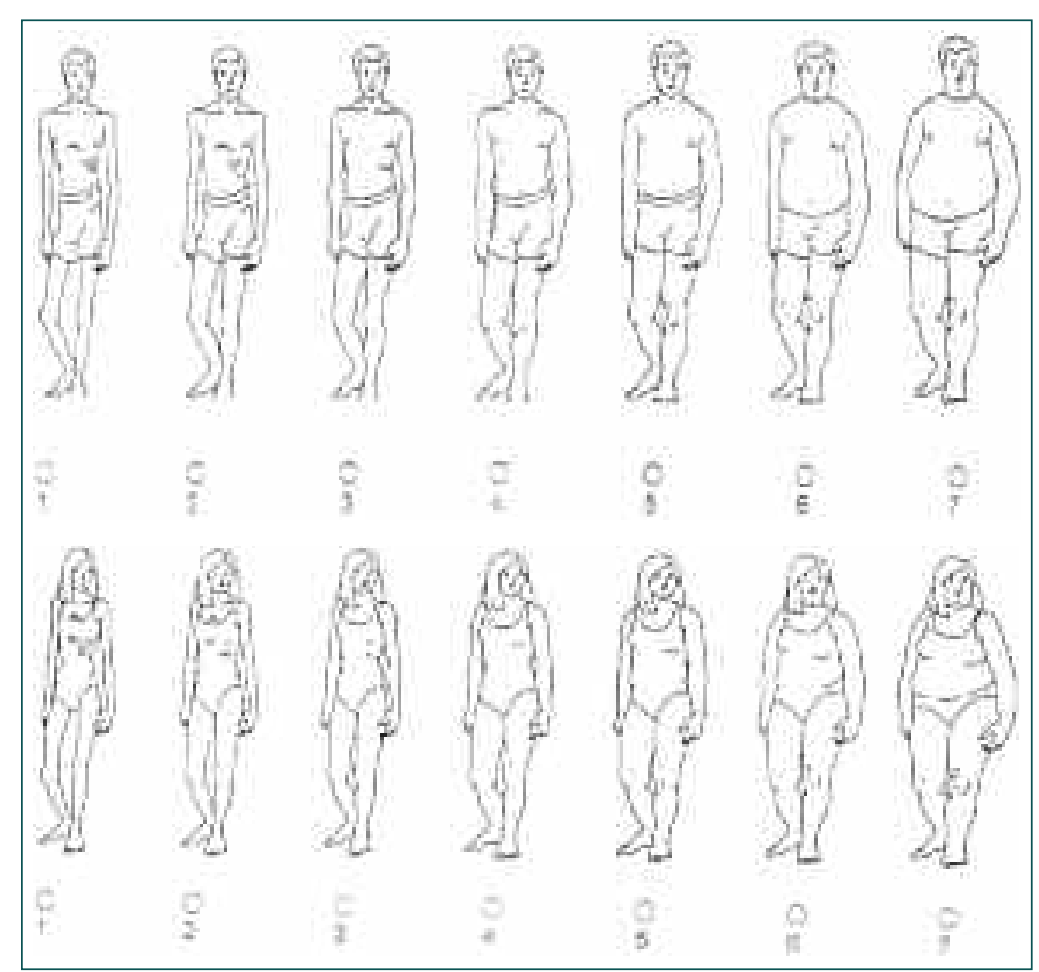

Figura 1. Classificação dos doentes por classes de obesidade. 
rexia Nervosa a pessoa não recorre regularmente a ingestão compulsiva de alimentos nem a purgantes (por exemplo, indução de vómito ou abuso de laxantes, diuréticos ou enemas).

- Tipo Ingestão Compulsiva/Purgativo: durante o episódio actual de Anorexia Nervosa, a pessoa tem comportamentos bulímicos ou purgativos (indução de vómitos ou abuso de laxantes, diuréticos ou enemas). No entanto, os Critérios Diagnósticos da DSM-IV não podem ser inteiramente aplicáveis à população mais jovem e a sua aplicação formal pode mesmo atrasar o início de uma intervenção, o que não é desejável.

Dentro deste espírito, a Society for Adolescent Medicine publicou em 1995 um Position Paper sobre a adaptação dos critérios diagnósticos de Anorexia Nervosa à população adolescente. ${ }^{10}$ No critério A, terá de ser tida em consideração a grande variabilidade na taxa, timing e magnitude do ganho em altura e peso durante uma puberdade normal. Enquanto o adulto tem já um peso e estatura estáveis, o adolescente está em crescimento, pelo que défices nutricionais graves podem ocorrer na ausência de uma perda de peso importante.

O critério C fica condicionado pela menor capacidade em elaborar conceitos abstractos que caracteriza esta idade, com consequentes implicações a nível do auto-conceito, motivação em perder peso e estados afectivos.

No critério $D$, temos de ter em consideração a ausência de períodos menstruais nas adolescentes mais jovens, o eventual atraso da menarca em situações de restrição alimentar e a incapacidade de prever a periodicidade dos períodos menstruais nos dois anos que se seguem à menarca.

\section{Avaliação somática}

Deverá incluir a avaliação dos sinais vitais, inspecção da pele e mucosas (desidratação, acrocianose), tensão arterial, estádio pubertário, dados antropométricos, incluindo o Índice de Massa Corporal (IMC), avaliação cardiovascular e palpação abdominal.

\section{Meios Complementares de Diagnóstico}

Rotinas incluindo análise sumária de urina, função tiroideia, cortisol, colesterol total, função hepática, cálcio, magnésio, fósforo, estradiol, progesterona, LH, FSH, testosterona, amilase sérica, ECG, densitometria óssea (em doentes com peso baixo por um período su- perior a seis meses), eventual ecografia pélvica e ressonância magnética cerebral (alargamento ventricular relacionado com a malnutrição, o qual é reversível).

\section{Complicações}

Gerais: Sensação de fraqueza, sinais de malnutrição, lanugo, queda de cabelo, extremidades frias, pele seca e com tonalidade amarelada (hipercarotinemia).

Balanço hidroelectrolítico: Hipocaliemia, hiponatremia, alcalose hipoclorémica, incapacidade em concentrar a urina, diminuição da taxa de filtração glomerular, cetonúria.

Neurológicas: Apatia, dificuldade de concentração, alterações do humor, irritabilidade, convulsões, neuropatia periférica, atrofia cortical.

Cardiovasculares: Palpitações, tonturas, vasoconstrição periférica com acrocianose, tempo de reperfusão capilar aumentado, disritmia, hipotensão ortostática, alterações electrocardiográficas (prolongamento do intervalo QT, anomalia das ondas T, defeitos de condução).

Músculo-esqueléticas: Dores desencadeadas pelo exercício, diminuição da densidade óssea, hipotrofia muscular.

Endocrinológicas: Fadiga, intolerância ao frio, amenorreia, diminuição da libido, regressão das características sexuais secundárias, diabetes insípida, hipercortisolismo.

Hematológicas: Anemia, neutropenia, trombocitopénia.

Gastrointestinais: Atraso do esvaziamento gástrico, obstipação.

Na adolescência as complicações endocrinológicas revestem-se de particularidades únicas:

- Atraso ou interrupção no desenvolvimento pubertário;

- Atraso da maturação sexual;

- Atraso no aparecimento da menarca na adolescente pré-púbere;

- Potencial diminuição da velocidade de crescimento quando o seu início é anterior ao surto de crescimento;

- Baixa estatura definitiva (se a Anorexia Nervosa não for tratada antes da fusão das epífises);

- Redução no pico de massa óssea.

São múltiplos os factores implicados, nomeada- 
mente, o quadro de hipogonadismo hipotalâmico que se instala com alteração na regulação da GHRF. As gonadotrofinas regressam aos padrões de produção pré-pubertária com diminuição da produção de somatomedina. A diminuição da produção de estrogéneos, os níveis elevados de glicocorticoódes e a malnutrição, podem conduzir a osteopenia com risco de fracturas patológicas e, sobretudo, a um não atingimento do potencial pico de massa óssea.

\section{BULIMIA NERVOSA - CRITÉRIOS DE DIAGNÓSTICO ${ }^{9}$}

A. Episódios recorrentes de ingestão alimentar compulsiva. Um episódio é caracterizado por ambos os seguintes aspectos:

(1) ingestão, num período limitado de tempo (por ex., num período até duas horas) de uma quantidade de alimentos que é definitivamente superior à que a maioria das pessoas consumiria num período de tempo semelhante e nas mesmas circunstâncias. (2) sensação de perda de controlo sobre o acto de comer durante o episódio (por exemplo, um sentimento de incapacidade para parar de comer ou controlar a quantidade e qualidade dos alimentos).

B. Comportamento compensatório inadequado e recorrente, com o fim de prevenir o aumento de peso, tal como induzir o vómito; usar indevidamente laxantes, diuréticos, enemas ou outros medicamentos; jejum ou exercício físico excessivo.

C. A ingestão compulsiva de alimentos e os comportamentos compensatórios inadequados ocorrem ambos, em média, pelo menos duas vezes por semana em três meses consecutivos.

D. A auto-avaliação é indevidamente influenciada pelo peso e forma corporais.

E. A perturbação não ocorre exclusivamente durante os episódios de Anorexia Nervosa.

\section{Tipos:}

- Tipo Purgativo: durante o episódio actual de Bulimia Nervosa, a pessoa induz regularmente o vómito ou abusa de laxantes, diuréticos ou enemas.

- Tipo Não Purgativo: durante o episódio actual de Bulimia Nervosa, a pessoa usa outros comportamentos compensatórios inadequados, tais como jejum ou exercício físico excessivo, mas não induz o vómito nem abusa de laxantes, diuréticos ou enemas.

\section{Avaliação somática}

Deverá incluir, para além dos aspectos referidos na Anorexia Nervosa, a inspecção das glândulas salivares (hipertrofiadas em situações de indução frequente do vómito) e do esmalte dentário (erosão), e a pesquisa do sinal de Russell (calosidades a nível das articulações interfalângicas dos dedos das mãos).

\section{Complicações}

Alterações hidroelectrolíticas, dores abdominais, alterações da motilidade esofágica e gástrica, gastrite e esofagite, pancreatite aguda, síndrome da artéria mesentérica superior.

\section{Indicações para Internamento}

As indicações para internamento de um adolescente com perturbação do comportamento alimentar são várias, nomeadamente: falência do tratamento no ambulatório, recusa persistente em se alimentar, indução de vómito ou utilização compulsiva de laxantes, malnutrição severa (IMC inferior a 13), desidratação, alterações hidroelectrolíticas, disritmia, bradicardia, hipotensão, hipotermia, convulsões, insuficiência cardíaca, emergência psiquiátrica (ideação suicida, surto psicótico) e comorbilidades mentais que interfiram com o tratamento (depressão grave, perturbação obsessivo-compulsiva, disfunção familiar grave).

Pode nem sempre ser fácil fazer o diagnóstico diferencial entre Anorexia e Bulimia, sobretudo nas situações menos claras. No entanto, perante um quadro de Anorexia Nervosa, temos de ter magreza. Ademais, a Anorexia tem habitualmente um início mais precoce que a Bulimia, podendo ocorrer antes da menarca. No entanto, tipicamente, tem o seu início na fase média da adolescência. Apesar de ser muito mais frequente no sexo feminino, afecta também o sexo masculino (9:1). Raramente há uma procura de ajuda. Associa-se frequentemente a ansiedade, a depressão ou a uma perturbação obsessivo-compulsiva. Na ausência de intervenção precoce, o prognóstico é mau.

Na Bulimia o peso é normal, o início é mais tardio sendo muito rara antes da menarca. É mais frequente o pedido de ajuda e é muito rara no sexo masculino (30:1). Tem frequentemente um curso flutuante, está frequentemente associada a depressão, auto-mutilação e consumo de substâncias. Tem melhor prognósti- 
co que a Anorexia.

A categoria Perturbação do comportamento alimentar sem outra especificação, ${ }^{9}$ onde muitas vezes o adolescente se inscreve, serve para classificar perturbações que não satisfazem os critérios das perturbações específicas acima descritas. Exemplos:

1. Para o sexo feminino, todos os critérios de Anorexia Nervosa estão presentes excepto a amenorreia.

2. Todos os critérios de Anorexia Nervosa estão presentes e, apesar de uma perda de peso significativa, o peso encontra-se dentro dos valores normais.

3. Todos os critérios de Bulimia Nervosa estão presentes excepto que os episódios de ingestão compulsiva e os mecanismos compensatórios inapropriados ocorrem numa frequência inferior a duas vezes por semana, ou têm uma duração inferior a três meses.

4. Uso regular de comportamentos compensatórios inapropriados por uma pessoa de peso normal após a ingestão de pequenas quantidades de alimentos (por exemplo, indução de vómito após comer duas bolachas).

5. Mastigar e cuspir repetidamente, mas não engolir, grandes quantidades de alimentos.

6. Perturbação de ingestão alimentar maciça: episódios recorrentes de ingestão alimentar maciça na ausência dos comportamentos compensatórios inapropriados característicos de Bulimia Nervosa.

Em todas as situações, não devemos utilizar critérios demasiado rígidos que possam impedir o reconhecimento de uma Perturbação do Comportamento Alimentar nos seus estádios mais precoces. Tal atitude vai retardar o acompanhamento especializado em equipa multidisciplinar e influenciar negativamente o prognóstico.

\section{REFERÊNCIAS BIBLIOGRÁFICAS}

1. Fonseca H. Compreender os adolescentes: um desafio para pais e educadores. $5^{a}$ ed. Lisboa: Editorial Presença; 2008.

2. Neinstein LS, Kaufman FR. Normal physical growth and development. In: Neinstein LS. Adolescent Health Care: a practical guide. 4th ed. Philadelphia: Philadelphia, PA: Lippincott Williams \& Wilkins; 2002. p. 3-51.

3. Fonseca $\mathrm{H}$, Ireland $\mathrm{M}$, Resnick M. Familial correlates of extreme weight control behaviors among adolescents. Int J Eat Disord 2002 Dec; 32 (4): 441-8.

4. Collins ME. Body figure perceptions and preferences among preadolescent children. Int J Eat Dis 1991 March;10 (2): 199-208.

5. Fonseca H, Matos MG, Guerra A, Gomes-Pedro J. Are overweight adolescents at higher risk of engaging in unhealthy weight-control behaviours? Acta Paediatr 2009 May; 98 (5): 847-52.

6. Fonseca $\mathrm{H}$, Matos MG. Perception of overweight and obesity among Portuguese adolescents: an overview of associated factors. Eur J Pub Health 2005 Jun; 15 (3): 323-8.

7. Fonseca H, Matos MG, Guerra A, Gomes-Pedro J. How much does overweight impact the adolescent developmental process? Child Care Health Dev 2011 Jan; 37 (1): 135-42.

8. Patton GC, Johnson-Sabine E, Wood K, Mann AH, Wakeling A. Abnormal eating attitudes in London schoolgirls: a prospective epidemiological study: outcome at twelve-months follow-up. Psychol Med 1990 May; 20 (2): 383-94.

9. American Psychiatric Association. DSM-IV-TR Manual de Diagnóstico e Estatística das Perturbações Mentais. $4^{\mathrm{a}}$ ed. Lisboa: Climepsi Editores; 2002.

10. Kreipe RE, Golden NH, Katzman DK, Fisher M, Rees J, Tonkin RS, et al. Eating disorders in adolescents: a position paper of the Society for Adolescent Medicine. J Adolesc Health 1995 Jun; 16 (8): 476-9.

\section{CONFLITOS DE INTERESSE}

A autora não tem qualquer conflito de interesse.

\section{ENDEREÇO PARA CORRESPONDÊNCIA}

Helena Fonseca

Departamento da Criança e da Família, Hospital de Santa Maria, Av. Prof. Egas Moniz, 1, 1649-028 Lisboa.

E-mail: helenaregalofonseca@gmail.com

Recebido em 29/12/2010

Aceite para publicação em 16/04/2011

\section{ABSTRACT}

\section{BEHAVIOURAL EATING DISORDERS IN ADOLESCENTS}

The purpose of the present paper is to examine the ways in which the epidemiology and diagnosis, medical complications, nutritional concerns, psychological issues, treatment and outcome for adolescents with eating disorders differ from those of adults, with particular emphasis on prevention and on the medical and developmental issues unique to this period.

Keywords: Eating Disorders; Adolescent; Prevention and Control. 